\title{
Alkali leaching control of construction and demolition waste based geopolymers
}

\author{
Dimitris Kioupis, AggelikiSkaropoulou, Sotiris Tsivilis and GlikeriaKakali \\ National Technical University of Athens, School of Chemical Engineering, Athens, Greece
}

\begin{abstract}
This study regards the synthesis of waste brick geopolymers and the evaluation of their alkali leaching potential. Geopolymers with varied alkali to $\mathrm{Al}$ molar ratios were prepared and their alkali leaching potential was measured. In order to improve the efflorescence of the waste brick geopolymers, $\mathrm{KOH}$ was used instead of $\mathrm{NaOH}$ in the activation solution, $\mathrm{Ca}$ cations were incorporated through the partial substitution of the brick powder by ground granulated blast furnace slag or extra $\mathrm{Al}$ was introduced in the activation solution. The results showed that the leachability of the products is close related to the alkali content of the activation solution while the use of $\mathrm{K}$ ions in the starting mixtures reduced the leachability potential. The same effect was observed in the case of the extra $\mathrm{Al}$ ions incorporation. The partial substitution of the wastes bricks by slag showeda slight increase of leachability even though denser materials with enhanced strengths were prepared.
\end{abstract}

\section{Introduction}

The Construction and Demolition Wastes (CDWs) comprise one of the heaviest and most voluminous solid wastes worldwide generated by the construction industry [1]. A major environmental issue associated with the CDWs is their landfill disposal. A way to release the environmental pressure is by recycling and reusing these materials [2]. Due to the high $\mathrm{SiO}_{2}$ and $\mathrm{Al}_{2} \mathrm{O}_{3}$ content of many CDWs (bricks, tiles concrete, ceramics etc), they can be utilized in the production of alkali activated materials or geopolymers $[3,4]$. Geopolymers are a class of inorganic materials which possess a three-dimensional polymeric-type structure. They are formed by the reaction of an alkaline solution with an aluminosilicate material at ambient or slightly elevated temperature [5].

The alkalis play a dual role in the geopolymerization process. First of all, they generate a high $\mathrm{pH}$ solution by releasing hydroxyl ions in order to dissolve the aluminosilicate precursors and to produce small reactive silico and alumino species. Furthermore, the alkali metal cations offer electroneutrality in geopolymers framework by balancing the negative charge in aluminum tetrahedrals. The high amounts of alkalis used in the geopolymers mixes and the weak binding of the alkaline metal cations ( $\mathrm{Na}$ or $\mathrm{K}$ ) with geopolymeric matrix lead to products which often suffer from severe efflorescence, when excess alkali oxide remains unreacted in the pore network. The efflorescence influences negatively the appearance of the products and can restrict the variety of geopolymers applications.

Several attempts have been made to reduce the efflorescence of geopolymers either by incorporating an
Al-rich [6,7] or Ca-rich source [6,8]or by using potassium hydroxide instead of sodium hydroxide in the activation solution [9]. In addition, hydrothermal curing effectively promote the geopolymerization reactions resulting in less unbound alkali which is able to mitigate efflorescence $[6,8]$.

In this study, geopolymers were synthesized from waste brick and their alkali leachability was evaluated. The effect of the alkali type ( $\mathrm{Na}$ or $\mathrm{K}$ ) and alkali content of the activation solution on the alkali leaching was measured. Then, Al-rich and $\mathrm{Ca}$-rich sources in the form of $\mathrm{Al}_{2} \mathrm{O}_{3}$ colloidal dispersion in $\mathrm{H}_{2} \mathrm{O}$ and ground granulated blast furnace slag were incorporated in geopolymer synthesis, respectively, in order to control the alkali leaching of brick waste geopolymers. XRD and FTIR were applied to characterize the products. The mechanical strength of the produced specimens was measured through compressive strength tests.

\section{Experimental}

Waste bricks (WB) collected from various demolished buildings of Attica (Greece), were used as the raw material. In some cases, ground granulated blast furnace slag (GGBFS) was added in the starting materials in order to improve the alkali leaching of the final products.Both materials were pulverized in order to obtain a certain fineness $\left(d_{50}=20 \mu \mathrm{m}\right)$ prior to their application. Table 1 shows the chemical composition of the WB and GGBFS in the form of oxides and trace elements, as determined by using X-ray fluorescence spectrometry (XRF). 
Table 1. The chemical composition of the raw materials (\% w/w).

\begin{tabular}{lcc}
\hline Oxide & WB & GGBFS \\
\hline $\mathbf{S i O}_{\mathbf{2}}$ & 51.31 & 39.49 \\
$\mathbf{A l}_{\mathbf{2}} \mathbf{O}_{\mathbf{3}}$ & 14.62 & 10.14 \\
$\mathbf{F e}_{\mathbf{2}} \mathbf{O}_{\mathbf{3}}$ & 8.45 & 0.89 \\
$\mathbf{C a O}$ & 6.33 & 39.97 \\
$\mathbf{M g O}$ & 8.10 & 6.75 \\
$\mathbf{K}_{\mathbf{2}} \mathbf{O}$ & 2.28 & 0.30 \\
$\mathbf{N a}_{\mathbf{2}} \mathbf{O}$ & 0.57 & 0.00 \\
$\mathbf{S O}_{\mathbf{3}}$ & 0.44 & 0.49 \\
$\mathbf{T i O}_{2}$ & 0.66 & - \\
$\mathbf{P}_{\mathbf{2}} \mathbf{O}_{\mathbf{5}}$ & 0.08 & - \\
$\mathbf{C l}$ & 0.04 & - \\
$\mathbf{L . O . I}$ & 3.97 & 0.16 \\
\hline
\end{tabular}

The Al and Si leaching of the WB was conducted by mixing $0.5( \pm 0.0001) \mathrm{g}$ of solid with $20 \mathrm{ml}$ of alkaline solution under continuous stirring. The concentration of the alkaline solution and the dissolution time were kept at $10 \mathrm{M}$ and $24 \mathrm{~h}$, respectively [10]. After filtering, the liquid part was diluted to $250 \mathrm{ml}$, the $\mathrm{pH}$ was adjusted to $\mathrm{pH}<1$ by adding concentrated $\mathrm{HCl}$ acid and Atomic Absorption Spectroscopy (AAS) was used in order to determine the $\mathrm{Al}$ and $\mathrm{Si}$ concentrations.

The alkaline solutions were prepared by dissolving $\mathrm{NaOH}(>99 \%$, CAS: $1310-73-2)$ or $\mathrm{KOH}(86 \%$, CAS: 1013-58-3) anhydrous pellets in distilled water and adding soluble $\mathrm{Si}$ in the form of $\mathrm{SiO}_{2}\left(50 \%\right.$ in $\mathrm{H}_{2} \mathrm{O}$, colloidal dispersion, CAS: 7631-86-9)and in some cases, soluble $\mathrm{Al}$ in the form of $\mathrm{Al}_{2} \mathrm{O}_{3}\left(20 \%\right.$ in $\mathrm{H}_{2} \mathrm{O}$, colloidal dispersion, CAS:1344-28-1). The activation solutions were stored for a minimum of $24 \mathrm{~h}$ prior to use, to allow equilibrium. Then, the starting materials (WB and / or GGBFS)and the activation solution were mechanically mixed (standard mortar mixer: Controls 65-L0005) to form a homogenous slurry which was transferred to 50x50x50 mm cubic molds and mildly vibrated. The specimens were left at room temperature for $2 \mathrm{~h}$ and then were cured at $70^{\circ} \mathrm{C}$ for $48 \mathrm{~h}$. Compression tests were carried out on a Toni-technik uniaxial testing press, 7 days after the specimens' preparation (load rate $1.5 \mathrm{kN} / \mathrm{s}$, according to the EN196-1 requirements). For each synthesis, three specimens were prepared and tested under compression.Mixtures were prepared with molar ratios as given in Table 2 .

Table 2.Chemical composition of the produced geopolymers.

\begin{tabular}{lcccccc}
\hline Mix & $\begin{array}{c}\text { WB } \\
(\boldsymbol{\%})\end{array}$ & $\begin{array}{c}\text { GGBFS } \\
(\boldsymbol{\%})\end{array}$ & $\begin{array}{c}\text { Alkali } \\
(\mathbf{R})\end{array}$ & Si/Al & R/Al & s/l \\
\hline B1 & 100 & 0 & $\mathrm{Na}$ & 3.5 & 0.1 & 2.4 \\
B2 & 100 & 0 & $\mathrm{Na}$ & 3.5 & 0.5 & 3.6 \\
B3 & 100 & 0 & $\mathrm{Na}$ & 3.5 & 1.0 & 3.6 \\
B4 & 100 & 0 & $\mathrm{Na}$ & 3.5 & 1.5 & 2.8 \\
B5 & 100 & 0 & $\mathrm{~K}$ & 3.5 & 1.0 & 3.6 \\
B6 & 100 & 0 & $\mathrm{Na}$ & 2.9 & 1.0 & 2.1 \\
B7 & 95 & 5 & $\mathrm{Na}$ & 3.5 & 1.0 & 3.6 \\
B8 & 90 & 10 & $\mathrm{Na}$ & 3.5 & 1.0 & 3.6 \\
\hline
\end{tabular}

The chemical composition of the mixtures was varied by changing the $\mathrm{Si} / \mathrm{Al}$ molar ratio of the starting mixture, the overall $\mathrm{Na} / \mathrm{Al}$ molar ratio, the solids to liquids mass ratio (s/l), the GGBFS content (\%) and the alkali type (R: $\mathrm{Na}$ or $\mathrm{K})$.

In order to determine the alkali leaching ability of the mixes, a modification of the experimental technique developed by Vafaeiet al. [7] which quantifies the free alkali content (alkali which is not structurally bonded) was applied. For this test, each geopolymer samplewas pulverized and passedthrough a $90 \mu \mathrm{m}$ sieve.1.0000 $( \pm 0.0001) \mathrm{g}$ of the obtained geopolymer was added to 40 $\mathrm{ml}$ of deionized water and stirred for $24 \mathrm{~h}$ to leach freealkali from geopolymerbinder. The solution wasthen filtered to remove solids and titratedwith $0.1 \mathrm{~N}$ hydrochloric acid. The $\mathrm{pH}$ and electrical conductivity of the filtrate was measured. The free alkali content of the geopolymer was calculated by the volume of the acid used.

The phase distribution of the powders was investigated by X-ray diffraction (XRD), on a Bruker D8 ADVANCE $x$-ray diffractometer. The data were collected in a $2 \theta$ range $2-70^{\circ}$, with $0.01^{\circ}$ step size and $1 \mathrm{sec}$ per step and were evaluated using Diffrac.Eva v3.1 software.FTIR measurements were carried out using a Fourier Transform IR Spectrophotometer (Jasco 4200 Type A). The FTIR spectra were obtained by the $\mathrm{KBr}$ pellet technique, in the wavenumber range from 400 to $4000 \mathrm{~cm}^{-1}$ and resolution $4 \mathrm{~cm}^{-1}$. The pellets were prepared by pressing a mixture of the sample and dried $\mathrm{KBr}$ (sample: $\mathrm{KBr}$ equals to $1: 200$, approximately) at 7.5 $\mathrm{t} / \mathrm{cm}^{2}$.

\section{Results and discussion}

\subsection{Extent of waste brick dissolution}

Table 3 presents the concentration of $\mathrm{Al}$ and $\mathrm{Si}$ in the solutions (10M NaOH or $\mathrm{KOH})$ of the WB. The results of the alkaline dissolution experiments showed that WB is susceptible to alkaline attack and, therefore, possess good geopolymerization potential. The degree of dissolution is higher in $\mathrm{NaOH}$ than in $\mathrm{KOH}$ solution which is in well accordance to the literature [11]. Due to their higher mobility, sodium ions show a higher tendency to attack and dilute aluminosilicon species of the raw material. However, it must be noted, that the leaching behavior of $\mathrm{Al}$ and Si cannot predict by itself the quality of the produced geopolymers. Further experiments concerning the preparation and properties of geopolymers from these raw materials have to be conducted.

Table 3.Extent of $\mathrm{Al}$ and $\mathrm{Si}$ dissolution in relation to alkali metal selection (ppm).

\begin{tabular}{lcc}
\hline Solution & \multicolumn{2}{c}{ CDW brick } \\
\hline & Si $(\mathbf{p p m})$ & Al $(\mathbf{p p m})$ \\
$\mathbf{1 0}$ M NaOH & 37.1 & 14.66 \\
$\mathbf{1 0}$ M KOH & 29.3 & 9.69 \\
\hline
\end{tabular}

\subsection{Alkali leachability tests}

Table 4 presents the free alkali content (wt. \%), the $\mathrm{pH}$ and the electrical conductivity of the filtrates as well as 
the compressive strength measured for the corresponding WB geopolymers.

Table 4.Free alkali content, $\mathrm{pH}$ and electrical conductivity of the leachates and the compressive strength of the corresponding samples.

\begin{tabular}{lcccc}
\hline Mix & $\begin{array}{c}\text { Free alkali } \\
\text { (\% wt.) }\end{array}$ & pH & $\begin{array}{c}\text { Electr. Cond. } \\
(\mathbf{m S})\end{array}$ & $\begin{array}{c}\text { Compr. Str. } \\
(\mathbf{M P a})\end{array}$ \\
\hline B1 & $4 \%$ & 8.8 & 0.5 & 0.42 \\
B2 & $23 \%$ & 10.0 & 2.0 & 7.15 \\
B3 & $26 \%$ & 10.4 & 3.4 & 16.37 \\
B4 & $28 \%$ & 10.6 & 4.4 & 1.92 \\
B5 & $23 \%$ & 10.3 & 3.8 & 20.22 \\
B6 & $21 \%$ & 10.4 & 2.7 & 3.96 \\
B7 & $28 \%$ & 10.4 & 3.5 & 16.84 \\
B8 & $28 \%$ & 10.8 & 3.3 & 23.38 \\
\hline
\end{tabular}

Figure 1 shows, schematically, the free alkali content and the compressive strength of the WB geopolymers varying in their alkalinity level ( $\mathrm{Na} / \mathrm{Al})$. It is obvious that the gradual introduction of higher amounts of alkalis in the starting mixtures shows a higher extent of alkali leaching, indicating a stronger tendency towards efflorescence. This is related to pore solution chemistry where the higher $\mathrm{Na} / \mathrm{Al}$ molar ratios generate excess of $\mathrm{Na}$ ions. These ions are left unboundfrom the geopolymer network and are trapped to the pores in the form of alkaline solutions.

The sample $\mathrm{B} 1(\mathrm{Na} / \mathrm{Al}=0.1)$ containa very low value of free alkali content $(4 \%)$. However, at the same time this sample possesses near zero compressive strength values $(0.42 \mathrm{MPa})$. It seems that the amount of alkali in the activation solution is too little to sufficiently solubilize the $\mathrm{Si}$ and $\mathrm{Al}$ species of the raw material, resulting in the delivery of a product which is not well geopolymerized.

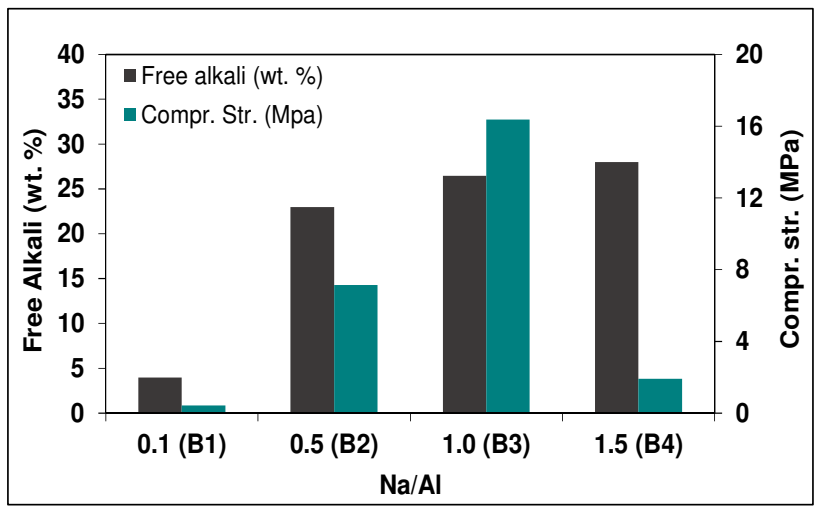

Fig. 1. Free alkali content and compressive strength of WB geopolymers with varying $\mathrm{Na} / \mathrm{Al}$ molar ratios.

In samples with $\mathrm{Na} / \mathrm{Al}>0.5$, the increase rate of the free alkali content seems to be minimized. This is attributed to the fact that higher $\mathrm{Na} / \mathrm{Al}$ molar ratios positively affect the extent of the geopolymerization reaction and therefore reduce the matrix permeability[12]. This is also reflected in the mechanical strength of the produced geopolymers which is enhanced.As an example, the sample B3 containing the two-fold amount $(\mathrm{Na} / \mathrm{Al}=$ 1.0) of alkalis in relation to sample $\mathrm{B} 2(\mathrm{Na} / \mathrm{Al}=0.5)$ possesses,considerably, higher mechanical strength (16.37 MPa) while the amount of free alkali is not significantly altered. However, this trend is not followed in the case of material $\mathrm{B} 4(\mathrm{Na} / \mathrm{Al}=1.5)$, which exhibits markedly reduced compressive strengths (1.92 MPa). For the preparation of the particular geopolymer, a large amount of water was required to make the paste workable, resulting in thepH reduction of the gel and therefore the limited dissolution of the WB. Furthermore, a higher porosity is createddue to the removal of the unbound water.

The results of alkali leachability and mechanical strength of the WB geopolymers showed that the highest compressive strength value is not necessarily combined with the lowest free alkali content. This indicates that there is a need to compromise between achieving the optimum mechanical behavior and controlling the unbound alkalis in the pore solutions in order to deliver a durable geopolymer.

Figure 2 presents the effect of the alkali type ( $\mathrm{Na}$ or $\mathrm{K})$ in the alkali leachability of the WB geopolymers. It is obvious that the incorporation of $\mathrm{K}$ ions to the gels favors the formation of high strength products with lower levels of free alkali content. Due to their greater size, $\mathrm{K}$ ions are not as mobile as the $\mathrm{Na}$ ions and thus create stronger bonding with the aluminosilicate gels, resultingin a lower alkali concentration in the pore solution which causes the efflorescence [13]. Regarding the mechanical behavior of the products, the $\mathrm{K}$ based sample exhibits enhanced compressive strength values by about $20 \%$ (20.22 MPa) in relation to the $\mathrm{Na}$ based product.

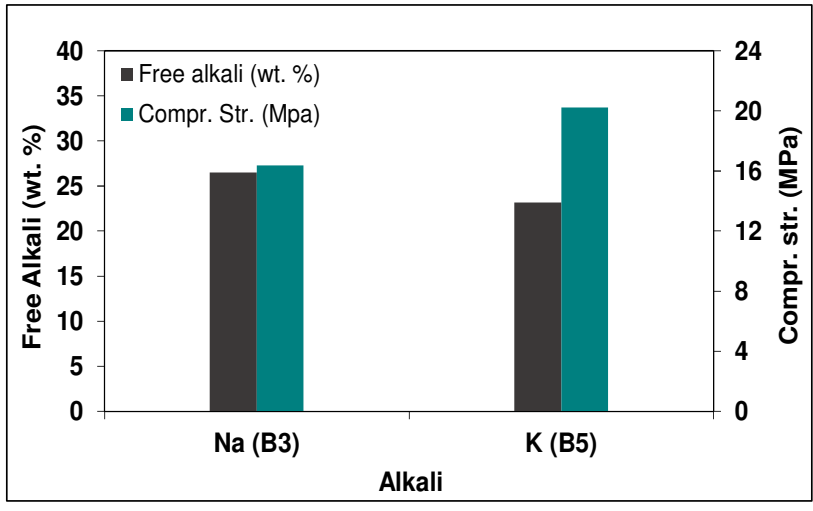

Fig. 2. Free alkali content and compressive strength of WB geopolymers with varying alkali type ( $\mathrm{Na}$ or $\mathrm{K}$ ).

As presented in Figure 3, the introduction of an $\mathrm{Al}$ rich source, such the one used in this study, led to the improvement of the alkali leachability of the WB geopolymers. The introduction of the additional Al helps the formation of a denser geopolymer molecular network which requires a larger number of $\mathrm{Na}$ cations to balance the negative charge of the 4-coordinated $\mathrm{Al}$ in the N-A-S$\mathrm{H}$ gels. This results in the promotion of the geopolymerization reaction and the delivery of a structure with a reduced free alkali content [7].

It was expected that the creation of a more stable geopolymeric network will deliver a product with enhanced mechanical strength. However, as it appears in this study, the introduction of aluminum had the opposite effect. This fact can be attributed tothe nature of the starting mixture of the sample B6. As in the case of B4, this mix required a great amount of water to make the 
paste workable resulting in an increased porosity which adversely affected the strength of the specimens.

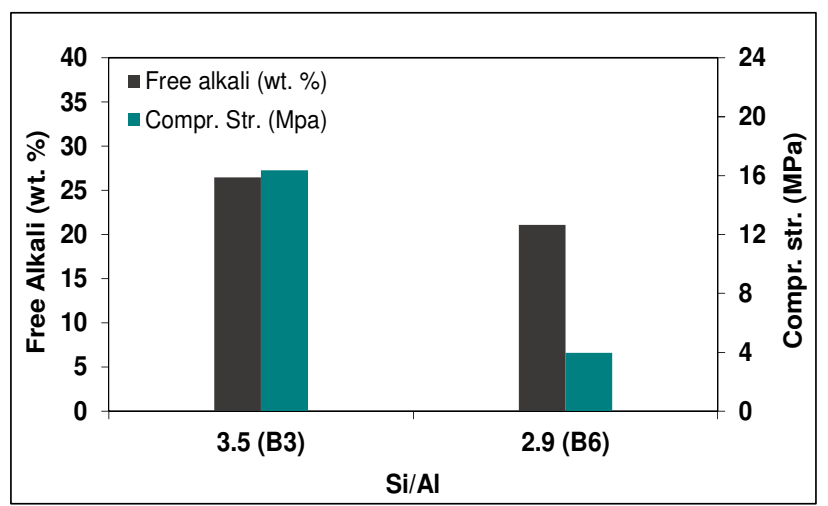

Fig. 3. Free alkali content and compressive strength of WB geopolymers with varying $\mathrm{Si} / \mathrm{Al}$ molar ratios.

Figure 4 presents the free alkali content and compressive strength of the samples with varying content of GGBFS.It is well understood that the introduction of considerable amounts of calcium in the geopolymerization system forms a homogenous and amorphous sodium calcium aluminosilicate hydrate (NC-A-S-H) networkin parallel to the sodium aluminosilicate hydrate (N-A-S-H) of the geopolymerization mechanism. The gels containing these extra phases lead to the formation of more compact microstructures which accountfor the high strength achieved in GGBFS containing geopolymers.

As it is observed from Figure 4,the introduction of low levels of GGBFS into the starting mixture does not promote the alkali leachability of the WB geopolymers. On the contrary, the incorporation of $\mathrm{Ca}$ ions in the geopolymer network lead to a slight increase of the free alkali content $(2 \%)$. This is related to the fact that the calcium ions act competitively to sodium ions in their connection to the aluminosilicate network in order to provide charge balance. The increased availability of sodium ions which remain unbound in the gels, contribute to the enhancement of the alkaline pore solution and therefore the free alkali content.

Even though the incorporation of GGBFS favors the formation of more compact structures with higher mechanical strength (e.g. B8 exhibits $23.38 \mathrm{MPa}$ ), the alkalinity of the pore solution is higher andthe capacity of pores to hold crystal deposits low.

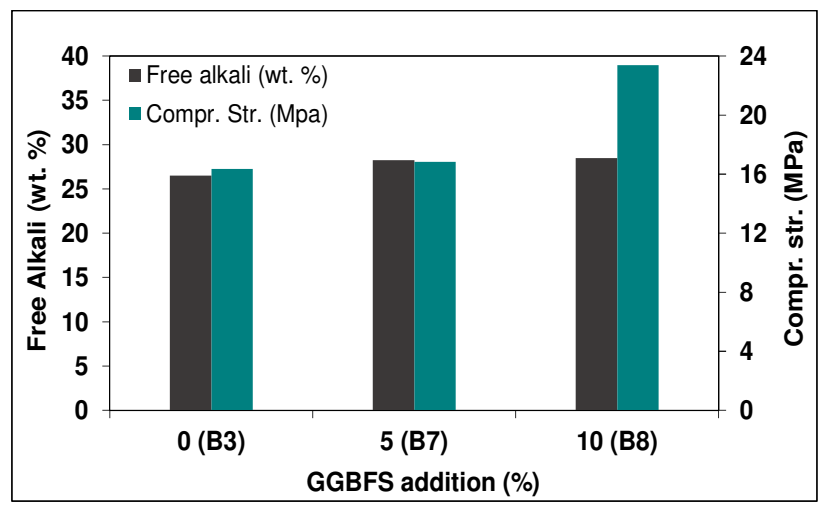

Fig. 4. Free alkali content and compressive strength of WB geopolymers with varying GGBFS addition.
As it seen from Table 4, a proportional relationship between the free alkali content and the values of $\mathrm{pH}$ and electrical conductivity is observed in almost all samples.

\subsection{Characterizations}

The XRD patterns of the $\mathrm{WB}$ and some representative geopolymers with varying $\mathrm{Na} / \mathrm{Al}$ molar ratios are presented in Figure 5. The main mineral phases in the WB are the quartz, albite, diopside, muscovite, microcline, maghemite and traces of carbonates (calcium or potassium). In the case of geopolymers (B2 and B4), the increase of the background, in the range $20-40^{\circ} 2 \theta$, indicates the formation of the amorphous geopolymer matrix. When the alkali content is high $(\mathrm{Na} / \mathrm{Al}=1.5)$, the formation of zeolites, such as chabazite, is favored and this is associated with lower compressive strength (Fig. 5, $\mathrm{B} 4)$. Besides, it is observed that the increase of $\mathrm{R} / \mathrm{Al}$ rate favors the enhancement of the alkali carbonates. The unbound alkalis react with the $\mathrm{CO}_{2}$ of the atmosphere and produces increased amounts of alkali carbonates. All other samples did not exhibit any differences in respect to their crystallographic phase content.

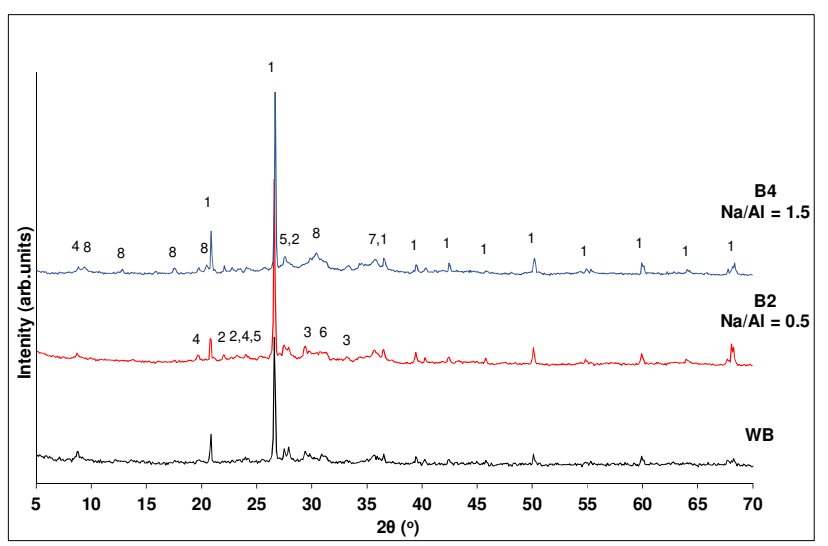

Fig. 5. XRD patterns of waste brick and selected geopolymers. 1: quartz, 2: albite, 3: carbonates, 4: muschovite, 5: microcline, 6: diopside, 7: maghemite and 8: chabazite.

Figure 6 shows the FTIR spectra of WB and selected geopolymer samples with varying $\mathrm{Na} / \mathrm{Al}$ molar ratios. The region of interest in both $\mathrm{WB}$ and geopolymer products lies in the range between 800 and $1300 \mathrm{~cm}^{-1}$. This broad hump exhibits overlapped peaks which are associated with the asymmetric stretching vibrations of $\mathrm{Si}-\mathrm{O}-\mathrm{T}$ (T: tetrahedral $\mathrm{Si}$ or $\mathrm{Al}$ ) bonds. In the case of the $\mathrm{WB}$, the maximum of this hump is at around $1110 \mathrm{~cm}^{-1}$ while in the case of the geopolymers this hump becomes narrower and shifts to lower wavenumbers $\left(\sim 1000 \mathrm{~cm}^{-1}\right)$. This alteration is directly linked with the substitution of $\mathrm{Si}$ by tetrahedral $\mathrm{Al}$ and therefore the formation of an aluminosilicate network [14]. Furthermore, the Si-O-T asymmetric bending vibrations at $\sim 460 \mathrm{~cm}^{-1}$ seems to be minimized with the formation of the aluminosilicate network. The band at $\sim 1635 \mathrm{~cm}^{-1}$ is related to $\mathrm{O}-\mathrm{H}$ bending vibrations of molecular water. All samples contain carbonate species pointed out by the presence of the band at around $1450 \mathrm{~cm}^{-1}$, related to anti-symmetric vibrations of $\mathrm{CO}_{3}{ }^{2-}$ ions. Samples with high R/Al ratios (B3 and B4) tend to exhibit more pronounced carbonate 
ion vibrations. The absorbance at $875 \mathrm{~cm}^{-1}$ is related to symmetric stretching vibrations of $\mathrm{Al}-\mathrm{O}$ and it constitutes sign of geopolymerization. As for 800,780 and $685 \mathrm{~cm}^{-1}$ peaks, these are connected with symmetric stretching vibrations of $\mathrm{Si}-\mathrm{O}-\mathrm{T}$ bonds.

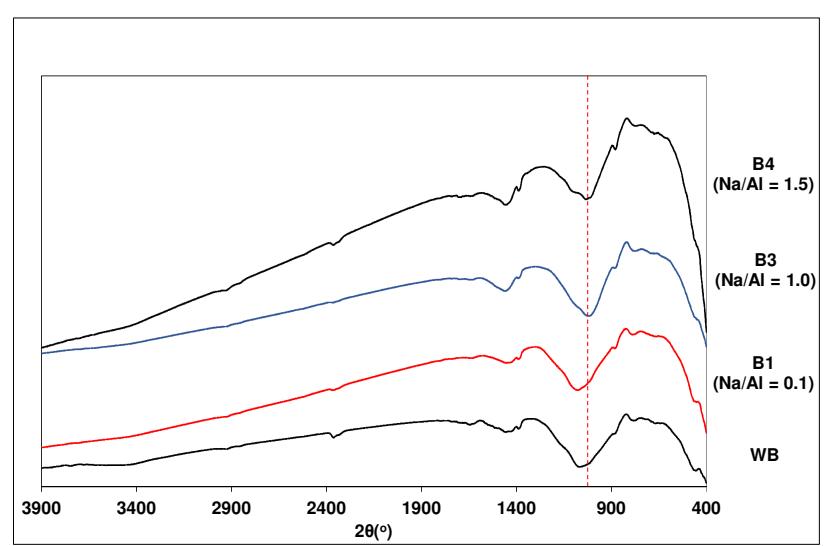

Fig. 6. FTIR spectra of WB and selected geopolymers.

\section{Conclusions}

This study presents an effort on controlling the alkali leachability of waste brick geopolymers which is responsible for the efflorescence phenomenon. The results showed that the alkalinity of the activation solution plays an important role on the free alkali content. Higher alkalinities tend to increase the leachability but at the same favor the mechanical strength of the products. It seems that the delivery of a durable waste brick geopolymer requires a compromise between the highest achievable mechanical strength and the lowest unbound alkali content. The selection of potassium ions favors the leachability of the products while at the same time promotes their mechanical behavior (20\%). The introduction of extra $\mathrm{Al}$ ions into the initial gels effectively reduces the free alkalissince more cross-linked geopolymer molecular networks are formed and thus larger amount of $\mathrm{Na}^{+}$cations is required to serve as charge balancing. Finally, the substitution of the waste brick by small amounts of ground granulated blast furnace slagenhanced the mechanical strength of the waste brick geopolymers but led to the aggravation of the alkali leachability. Further studies upon the combinational effect of the aforementioned approaches on the alkali leaching ability of the waste brick geopolymers are recommended in order to improve the durability of the products.

\section{Acknowledgments}

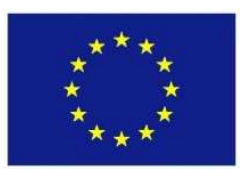

This project has received funding from the European Union's Horizon 2020 research and innovation programme under grant agreement No [723825].

\section{References}

1. European Commission (DG ENV), Final Report Task 2Service Contract on Management of Construction and Demolition Waste, 2011 (ENV.G.4/FRA/2008/0112).

2. M. Del Rio Merino, J. Navarro, P. Saez, Open Constr. Build. Technol. J. 5, 124 (2011)

3. K. Komnitsas, D. Zaharaki, A. Vlachou, G. Bartzas, M. Galetakis, Adv. Powder Technol.26, 368 (2015)

4. L. Reig, M.M. Tashima, M.V. Borrachero, J. Monz, C.R. Cheeseman, J. Pay, Constr. Build. Mater. 43, 98 (2013)

5. J. Davidovits, J. Ther. Anal. 37, 1633 (1991)

6. E.N. Kani, A. Allahverdi, J.L. Provis, Cem.Concr. Compos.34, 25 (2012)

7. M. Vafaei, A.Allahverdi,Adv. Powder Technol. 28,215 (2017)

8. Z. Zhang, J.L. Provis, A. Reid, H. Wang,Cem. Concr. Res.64,30 (2014)

9. P. Duxson, J.L. Provis, G.C. Lukey, J.S.J. van Deventer, F. Separovic,Z.H. Gan, Ind. Eng. Chem. Res.45,9208 (2006)

10. Ch. Panagiotopoulou, E. Kontori, Th. Perraki, G. Kakali, J. Mater. Sci. 42, 2967 (2007)

11. X.U. Hu, J.S.J. Van Deventer, Int. J. Miner. Process59, 247 (2000)

12. R.R. Lloyd, J.L. Provis, J.S.J. van Deventer, Cem.Concr. Res. 40,1386 (2010)

13. O. Bortnovsky,J. Dědecek, Z. Tvaruzkova, Z. Sobalík, J. Subrt, J. Am. Ceram. Soc. 1, 3052 (2008)

14. T. Bakharev,Cem. Concr. Res.35, 1224 (2005) 